\title{
Condiciones de trabajo y configuración del sentido de la profesión académica en profesores universitarios colombianos
}

\author{
Martha Patricia Romero Caraballo
}

\begin{abstract}
How to cite this article:
Romero, M.P. (2019). Working conditions and the meaning of the academic profession in colombian university professors. Acta Colombiana de Psicología, 22(2), 267-279. doi: http://www.doi.org/10.14718/ACP.2019.22.2.13
\end{abstract}

Recibido, noviembre 1/2017; Concepto de evaluación, febrero 22/2018; Aceptado, febrero 17/2019

\author{
Martha Patricia Romero Caraballo \\ Pontificia Universidad Javeriana, Cali, Colombia. \\ ORCID: https://orcid.org/0000-0003-4018-3350
}

\begin{abstract}
Resumen
Enmarcada en el contexto de las transformaciones del trabajo académico, en especial con la irrupción del capitalismo académico, y situada en las organizaciones universitarias, la presente investigación tuvo como objetivo analizar la configuración del sentido de la profesión académica en 160 profesores universitarios pertenecientes a universidades públicas y privadas de cinco ciudades colombianas. Este estudio fue cuantitativo, no experimental, transversal y descriptivo. Se aplicó el instrumento QPW-5 -cuestionario sobre la calidad de vida laboral en organizaciones de servicios humanos, compuesto por 173 ítems que miden las escalas de condiciones de trabajo y bienestar laboral general-, el inventario de Burnout de Maslach, y un cuestionario de engagement, además de una parte cualitativa con cinco palabras clave que definen la experiencia de trabajar. Se realizó un análisis correlacional y un análisis de componentes principales para establecer el patrón de las dimensiones evaluadas. Como resultados, se encontró que los docentes encuestados evidenciaron tendencias generales a configurar un sentido de la profesión académica de forma ambivalente, en un polo positivo y negativo, donde se destaca la presencia de condiciones de trabajo fragmentadas que denotan agotamiento, cinismo y efectos colaterales, así como una nueva configuración del profesionalismo académico. Además de esto, se señala que la temporalidad del vínculo contractual se naturaliza como condiciones de trabajo precarias que colocan como foco de atención a los riesgos psicosociales de los docentes universitarios.

Palabras clave: sentido de la profesión, condiciones de trabajo, académicos, profesionalismo.
\end{abstract}

\section{Working conditions and the meaning of the academic profession in Colombian university professors}

\begin{abstract}
The aim of this research was to analyze the shaping of the meaning of the academic profession in 160 scholars, from public and private universities in four Colombian cities. The instrument QPW-5 (Questionnaire on Quality of Work life in Human Service Organizations) was applied. It was composed of 173 items distributed in five scales: Working Conditions, Perceived Workload, General Working Well-being, Maslach Burnout Inventory and Engagement, and five key words that define the work experience. This study was carried out following a quantitative approach, a non - experimental design with a cross-sectional descriptive correlation. A correlational analysis and Principal Component Analysis were performed to establish the pattern of the dimensions evaluated. The teachers surveyed showed general tendencies to shape their meaning of the academic profession in an ambivalent manner in a positive and negative pole, where the presence of fragmented working conditions that denote exhaustion, cynicism and collateral effects, as well as a new configuration of academic professionalism are highlighted. It is pointed out that the temporality of the contractual relationship is naturalized as a condition of precarious working conditions, placing a focus on the psychosocial risks of university teachers.

Key words: sense of the profession, working conditions, academics, professionalism.
\end{abstract}

$\mathrm{PhD}$, docente investigadora de la Pontificia Universidad Javeriana, Cali, calle 18 n. ${ }^{\circ} 118-250$. Tel.: +57 2 3218200, ext.: 9060 . Grupo de Investigación Bienestar, Cultura y Sociedad, BITACUS. martha.romero@javerianacali.edu.co 


\title{
Condiçóes de trabalbo e configuraçáo da percepçáo da profissáo acadêmica em professores universitärios colombianos
}

\begin{abstract}
Resumo
Enquadrada no contexto das transformações do trabalho acadêmico, em especial com a explosão do capitalismo acadêmico, e situada nas organizações universitárias, a presente pesquisa teve como objetivo analisar a configuração da percepção da profissão acadêmica em 160 professores universitários pertencentes a universidades públicas e privadas de cinco cidades colombianas. Este estudo foi quantitativo, não experimental, transversal e descritivo. Aplicou-se o instrumento QPW-5 — questionário sobre a qualidade da vida laboral em organizações de serviços humanos, composto por 173 itens que medem as escalas de condições de trabalho e bem-estar laboral geral—, o inventário de Burnout de Maslach e um questionário de Engagement, além de uma parte qualitativa com cinco palavras-chave que definem a experiência de trabalhar. Realizou-se uma análise correlacional e uma análise de componentes principais para estabelecer o padrão das dimensões avaliadas. Como resultado, descobriu-se que os docentes participantes evidenciaram tendências gerais para configurar uma percepção da profissão acadêmica de forma ambivalente, em um polo positivo e negativo, no qual se destaca a presença de condições de trabalho fragmentadas que denotam esgotamento, cinismo e efeitos colaterais, bem como uma nova configuração do profissionalismo acadêmico. Além disso, indica-se que a temporalidade do vínculo contratual é naturalizada como condições de trabalho precárias que chamam a atenção para os riscos psicossociais dos docentes universitários.

Palavras-chave: percepção da profissão, condições de trabalho, acadêmicos, profissionalismo.
\end{abstract}

\section{Introducción}

\section{El trabajo académico y sus transformaciones}

Históricamente, desde el surgimiento de las universidades en la época medieval -donde se caracterizaban por la exclusividad de la educación para ciertas comunidades de índole religioso (Brunner,1990, 2014; Universia, 2015)-, hasta la época de la modernidad ilustrada, industrial y democrática -donde la universidad funciona como una institución social (Blanch, 2013b)-, y al adoptar modelos en donde preponderan valores como la universalidad, la autonomía, la libertad, la racionalidad y la sabiduría, entre otros (Universia, 2015), las universidades han pasado por una serie de transformaciones que han permeado de manera significativa el campo administrativo y profesoral de las instituciones de educación superior (Romero, 2015).

Según Morales (2012), algunas de las transformaciones se han evidenciado con el desarrollo de la globalización -luego de la crisis del fordismo en los años setenta del siglo $\mathrm{XX}-$, y una de ellas es la introducción de las lógicas del mercado -en concordancia con el paradigma empresarial-, a partir de la cual se adoptan los criterios de productividad, rentabilidad, eficiencia, eficacia, competitividad y evaluación de competencias, resultados y relaciones laborales (Blanch, 2011b, 2014; Spicer \& Flemming, 2007).

Además de esto, según Chomsky (2014), dichas transformaciones generan tanto un cambio en el estilo de contratación, el cual pasa a ser de estable a temporal -e, incluso, precario-, donde uno de los aspectos que se ve más afectado es la desaparición de la carreara académica; adicional a que la proporción de personal administrativo se torna desproporcionada respecto a la población de profesores debido a que aumentan los cargos administrativos respecto a las plazas académicas. Teniendo esto en cuenta, parece que en las universidades se presenta un estilo empresarial burocrático que obliga a la creación de estamentos para el cumplimiento de trámites administrativos y educativos (Antunes \& Alves, 2004); además de una desproporción en la cobertura, el control de clases y la atención a los estudiantes - por el incremento del número de alumnos por clase-, un aumento en el número de profesores temporales, y una sobrecarga de tareas de índole administrativo y académico (Blanch, 2011a, 2013b, 2014; Calera, Esteve, Roel \& Uberti-Bona, 2014; Sánchez \& Del Sagrario, 2012).

Cabe mencionar que dichos cambios en las condiciones laborales de los académicos se presentan debido a reformas de corte neoliberal, en donde se pasa de tener un modelo keynesiano-fordista a instaurar un modelo que moderniza los servicios públicos -en el cual cabe la educación superior (Romero, 2015)-; con un nuevo paradigma, denominado New Public Mangement (o Nuevo Gerencialismo Público, NGP), en el que las organizaciones públicas son controladas y diseñadas por programas que deben ser medidos según sus resultados (Arellano \& Cabrero, 2005; Sisto, 2012; Sisto \& López, 2014).

Específicamente, el proceso del NGP es la puesta en marcha del modelo de gestión regido por la flexibilización, en donde se destacan la calidad, la excelencia, la innovación, la gobernanza y la sostenibilidad; proceso que se 
logra con la realización de evaluaciones por competencias y resultados -control de gastos y aplicación de indicadores costo-beneficio-, lo que trae como consecuencia que las instituciones públicas -sociales, sanitarias y educativasdeban asumir las reglas de libre comercio, donde quien recibe el servicio es visto solo como un cliente (Blanch, 2013b). En esta misma lógica, la NGP desafía los modos en los que se ha construido el vínculo entre empleador (universidad) y empleado (profesor), por lo que se genera entonces una nueva relación en la que, además de cambios en los salarios y la estabilidad laboral, se transforman los estándares de cumplimiento de la labor académica (Sisto, 2012). En otras palabras, si no se cumple con lo establecido por los nuevos cánones estandarizados, el salario o cualquier otra condición laboral se verán afectados.

De este modo, en la nueva gestión de las instituciones educativas universitarias -vistas como una nueva forma de relación-, el trabajo académico se reorganiza al mismo tiempo en que se reconfiguran los centros docentes tanto en sus planes de estudio como en las ofertas de las titulaciones (Blanch, 2013a). Teniendo esto en cuenta, las nuevas condiciones de trabajo académico -regidas bajo características de un contrato flexible- se convierten en algo en lo que la inestabilidad y la intensificación del tiempo de trabajo son recurrentes, además de que se promueve una reducción en las condiciones satisfactorias de la calidad de vida laboral e, incluso, en el rendimiento de los académicos en sus funciones (Caballero \& Nieto, 2015; Morales \& Blanch, 2013; Romero, Blanch \& Rentería, 2016).

Adicional a esto, es importante tener en cuenta que las nuevas formas y condiciones de trabajo son asumidas de manera particular por parte del profesorado, $\mathrm{y}$, en este mismo sentido, Anderson (2008) propone una clasificación de las formas de afrontamiento de los académicos con respecto a las actitudes que tienen hacia estas nuevas condiciones: (a) la evitación, entendida como el no realizar las nuevas demandas que se les presenta a los académicos; (b) la resistencia, en la cual los académicos presentan actitudes de rechazo firme hacia el gerencialismo; (c) la debilidad académica, con la que se busca que no se tengan en cuenta las nuevas condiciones debido a los rendimientos bajos que se obtienen, pero que se logre mantener dentro de la actividad académica con un bajo perfil; (d) el cumplimiento de forma cualificada, con respecto a las tareas encomendadas y las nuevas que se han colocado; y (e) la negación, una de las formas de afrontamiento que se refleja en los olvidos ocasionales como forma de rechazo sutil hacia las nuevas demandas gerenciales. Estas formas de afrontamiento ante condiciones que producen malestar en los profesionales de la academia emanan de las diversas condiciones transformadas del ámbito universitario que tienden a apartar a los profesionales de su ideal primario de la promulgación de un conocimiento útil para otros (Sánchez \& Del Sagrario, 2012; Zamora-Fortuny, 2004).

Así, la nueva gestión de la universidad en Latinoamérica -en la que se enmarca la universidad en Colombia- ha afectado la forma en la cual fue concebida la educación superior, dado que inicialmente se creó bajo el manto proteccionista del Estado, con un régimen de autonomía y sin obligación de rendir cuentas ideológicas a elementos externos (Brunner, 1990, 2012, 2014). Según Brunner (2014), en la escena académica latinoamericana se hace referencia a lo público y a lo privado de las universidades, donde a las instituciones públicas se les confiere la potestad del interés por la investigación y el servicio gratuito, mientras que las privadas se ven más como una dependencia comercial que busca recompensa y reconocimiento; $y$, en este sentido, las universidades latinoamericanas están transitando por los dominios del mercado (oferta-demanda) como alternativa para la sostenibilidad, la recuperación de lo financiero y la búsqueda de una nueva identidad (Mollis, 2003; Romero, Blanch \& Rentería, 2016).

Bajo este panorama, que se refleja en la profesión académica, se observa, no solo a nivel de Latinoamérica, sino también en Colombia, que las universidades están enmarcadas en lo que se define como "capitalismo cognitivo" o “capitalismo académico" (Slaughter \& Leslie (1997, 2001), cuya principal premisa es establecer una nueva forma de gestionar la academia mediante nuevas prácticas en las Instituciones de Educación Superior (IES) que garanticen la generación y expansión de ingresos al emular conductas mercantilistas y convertirse en una relación de venta de productos y servicios con fines de autofinanciamiento.

Desde este punto de vista, al hacer funcionar espacios que están siendo diseñados como si fueran mercados, la necesidad de tener financiación de cualquier sector privado o público hace que los académicos replanteen su quehacer (Romero, 2015), en donde las condiciones laborales del personal docente y académico de las IES se perciben como una comercialización de la producción del conocimiento y de aspectos como la cultura, la misión, la visión, las políticas de las universidades y la valoración de la investigación; razón por la cual se producen cambios en las subculturas de académicos y estudiantes (Mendoza \& Berger, 2006). Por consiguiente, la lógica generalizada del capitalismo académico se comparte con la lógica de la industria, por lo que los temas académicos se relacionan con las fuerzas del mercado, y la contribución de la investigación al conocimiento se convierte en una aplicación comercial (Slaughter \& Rhoades, 2004).

Por otra parte, los efectos de este capitalismo académico se tornan variables según el contexto, ya que en Latinoamérica 
270

-y, en concreto, en Colombia- las reformas direccionadas a la actualización de la Educación Superior (ES) aún no terminan de fincarse. Por ejemplo, en Colombia, la transformación de las universidades se viene presentando de forma más explícita desde el año 1993, debido a que las universidades públicas deben asumir características del sector privado; aspectos que han sido revaluados tanto por los académicos como por los estudiantes (MEN, 2013). Así, la legislación nacional que compete a la ES en Colombia -como la ley 115 de 1994, la ley 30 de 1992 y los decretos 2904 de 1994, 1279 de 2002 y 2566 del 2003-, designan el derrotero de la ES, ya que las universidades han asumido formas diversas de administración, así como de asunción de políticas de consecución o atracción de estudiantes para los diferentes programas, además del desarrollo de la docencia y las nuevas políticas para el reconocimiento de la producción intelectual y el incentivo por la investigación (Romero, 2015).

Teniendo esta legislación en cuenta, las universidades tanto públicas como privadas edifican sus proyectos institucionales y educativos -alrededor de sus misiones-al definir principios, propósitos y estrategias que abarcan los campos de la docencia, la investigación y la proyección social propuestas en la misma (Rizo, 2004; Romero, Blanch \& Rentería, 2016).

Sin embargo, la influencia no ha sido solo de las reformas legislativas, sino también de cambios a nivel social que permean el contexto, tal como sucede con la globalización, las tecnologías de la información y la comunicación, y la flexibilidad laboral; características que generan en las IES la perdida de independencia laboral y la modificación de las formas de relación con los académicos, sobre todo debido a la transformación de las relaciones tecnócratas y a la mercantilización del conocimiento (Castro-Gómez \& Guardiola, 2002).

Por otra parte, las nuevas formas de trabajo académico implican también nuevas condiciones de trabajo, ya que se le demanda a los académicos su adaptación a los nuevos cánones de producción, razón por la cual deben resignificar: (a) sus relaciones con el nuevo contexto (Blanch, 2014; Ochoa \& Blanch, 2016; Romero, Blanch \& Rentería, 2016; Sisto, 2005, 2012); (b) los parámetros de medición de las competencias académicas -por méritos o por el aumento de la producción intelectual (MEN, 2013)- con el ánimo de mejorar las condiciones laborales, aunque con esto se genera la competitividad entre los académicos de la misma universidad o de otras IES (Blanch, 2013a, 2013b; Romero, 2015); (c) las vinculaciones laborales de índole temporal u ocasional para dar respuesta a la demanda en la matriculación de estudiantes por programas, los cuales deterioran la dedicación docente a las tareas propias de la academia -en especial, la investigación y la atención a estudiantes-, situación que se percibe como riesgo psicosocial por la inestabilidad laboral y la disminución de las condiciones de trabajo docente (Blanch, 2011a, 2011b, 2013a, 2013b; Romero, 2015); y (d) la fragmentación de las contrataciones en la población académica, al considerar a la profesión académica como una profesión de carrera (Sánchez \& del Sagrario, 2012; Zabalza, 2013).

Cabe mencionar que este fenómeno se puede explicar bajo dos aristas: la primera, por la fragmentación en las contrataciones, debido a que no generan continuidad en el ejercicio y formación profesoral (Prieto, Valencia, Carrillo \& Castellanos, 2011); y la segunda, por la combinación de tareas propias de la docencia e investigación con las tareas administrativas que son de carácter obligatorio (Sánchez $\&$ del Sagrario, 2012).

En palabras de Fernández y Pérez (2011), la profesión académica actual presenta una situación muy particular explicada en cuatro aspectos: (a) la masificación de la ES, que genera un mayor número de estudiantes matriculados en las facultades y que trae como consecuencia una disminución de las posibilidades de crear nuevos espacios de reflexión acerca del conocimiento; (b) la proliferación de diversos tipos de IES, que establece una configuración de la profesión académica con características diferenciadas y negativas para el sistema educativo dada la existencia de plazas que son ocupadas por individuos que no consideran la profesión académica como carrera, sino como un trabajo más por realizar; (c) una baja inversión en la investigación y el desarrollo, que limitan el presupuesto de la investigación y la formación de los académicos; y (d) los procesos de internacionalización y regionalización de las IES, en donde se desarrollan procesos de globalización e implementación de las TIC.

En conclusión, las condiciones de trabajo en la profesión académica han estado inmersas en una serie de transformaciones que han hecho que el sentido de esta se vea igualmente en transformación, o que se configure a partir de la relación que tiene con el contexto en el que está inmersa la universidad como institución educativa en el país (Romero, Blanch \& Rentería, 2016).

\section{Sentido de la profesión académica}

El sentido de la profesión académica es entendido, desde la postura de Romero, Blanch y Rentería (2016) como cierto "profesionalismo", en el cual aparece la centralidad de la relación entre profesor-estudiante -asumida como la vocación académica, donde el interés por un tercero es fuente de satisfacción-, y el establecimiento de un vínculo de responsabilidad, confianza y respeto que garantice el 
contrato social de la educación con la sociedad -direccionados al mantenimiento de estándares de calidad y de actualización del conocimiento que se comparte-.

Pero el grado de satisfacción no solo depende de la compatibilidad entre la profesión que se ha escogido como carrera respecto al tipo de cátedra impartida -es decir, al grado de desarrollo del conocimiento de la disciplina-, sino también a las condiciones y riesgos psicosociales en los que se presenta el trabajo que se realiza (Arenas \& Andrade, 2013; Blanch, 2013a, 2013b; Garrido, Uribe \& Blanch, 2011; Romero, 2015; Romero, Blanch \& Rentería, 2016). Una de estas condiciones, particular a la profesión académica, es la libertad de cátedra, un componente que matiza la relación profesor-estudiante como un elemento constitutivo de la satisfacción por el hecho de formar a otros en su área de experticia. Este ethos de la profesión da la posibilidad de nutrir la relación con aspectos críticos que dan la posibilidad de un pensamiento diferente (Isola, 2013).

Asimismo, el profesionalismo en la academia está encaminado en varias direcciones: una específicamente hacia el conocimiento bajo cánones éticos y morales; y otra, hacia la motivación intrínseca y extrínseca de trabajar en el ámbito educativo. Así, la motivación del profesorado hacia la profesión que se ejerce está referida tanto a la relación que se establece con los estudiantes, como con el hecho de mantener la vitalidad por realizar una tarea profesoral diversa que aporte y sea enriquecedora (elementos extrínsecos), y un deseo de desarrollo constante a nivel intelectual y profesional (elementos intrínsecos); elementos esenciales para el proceso de la formación universitaria (Eirín, García \& Montero, 2009).

Si bien el profesionalismo en la academia va direccionado a la enseñanza de forma ética en la relación profesorestudiante, esta se ve afectada por las transformaciones de las condiciones laborales en la educación superior (Nixon, Marks, Rowland \& Walker, 2010), ya que, por ejemplo, el aumento de estudiantes y la disminución de tiempo para realizar asesorías o las altas cargas de trabajo administrativo, entre otras condiciones que devienen de la nueva gestión de las universidades (Blanch, 2014; Guerrero, 2006; Sisto, 2005, 2009, 2012), han hecho que el sentido de la profesión académica (profesionalismo) no se presente de una misma forma, sino que tenga distintos matices, expresados como resistencia, acto de acople o moldeamiento a nuevas formas de medición, control y calidad de programas de gestión profesoral, investigación y administración (Evans \& Cosnefroy, 2013; Nixon et al., 2008; O'Reilly \& Reed, 2011; Romero, Blanch \& Rentería 2016).
Por otra parte, la construcción del sentido de la profesión académica se realiza según los fenómenos se presenten que al interior de la profesión, como las prácticas que se suscitan por las condiciones laborales, la naturaleza de la tarea, el compromiso y la satisfacción que se siente al realizarlas (Ardichvili \& Kuchinke, 2009; Hackman \& Oldham, 1974, 1975; Romero, Blanch \& Rentería, 2016); y esta construcción puede presentarse tanto en polos positivos -con lógicas de satisfacción y compromiso, o engagement (Salanova \& Schaufeli, 2004, 2009)- como negativos -donde las condiciones de trabajo son precarias, poco motivantes y tienen alto compromiso, pero hay una imposibilidad de responder o afrontar las demandas, lo que hace que la tarea académica sea asumida como un sinsentido o como un síndrome de Burnout (Alcántara \& Preciado, 2009; Romero, Blanch \& Rentería, 2016; Salanova, Schaufeli, Llorens \& Pieró, 2000) - que se desglosan de la organización (universidad) y en el trabajo propiamente dicho.

Si todos los niveles de análisis se encuentran en condiciones favorables, la configuración del sentido de la profesión tendrá entonces características similares a los marcos de referencia que los nutre (Spink \& Medrado, 2013), y, por consiguiente, será denotado como favorable, óptimo o positivo; pero, de no existir proporcionalidad, el sentido se configura a partir de cualquiera de los elementos que lo constituyen, y, por tanto, cualquiera que sea el nivel que tenga mayor grado de significancia, así mismo será el sentido que se configure de la profesión (Romero, 2015; Romero, Blanch \& Rentería, 2016) (véase Figura 1).

Por último, es importante mencionar que los cambios en las condiciones de trabajo en las universidades han ocasionado un debate acerca de la ocupación y las tareas que se desempeñan al interior de la profesión, en su mayoría, debido al aumento de tensiones en el papel de la universidad como institución reguladora de la educación superior (Romero, 2015). Así, la fragmentación en las condiciones laborales, los efectos colaterales manifestados por los profesionales de la academia y las nuevas formas de exploración del profesionalismo académico son algunos de los aspectos que se ven matizados por las transformaciones en el ámbito académico, donde la experiencia de socialización de los docentes universitarios con su trabajo hace que se configure el sentido de una profesión que es considerada como una forma de vida y un fin para la misma.

Teniendo todo lo anterior en cuenta, la presente investigación tuvo como objetivo analizar la configuración del sentido de la profesión académica y las condiciones laborales de profesores universitarios colombianos. 


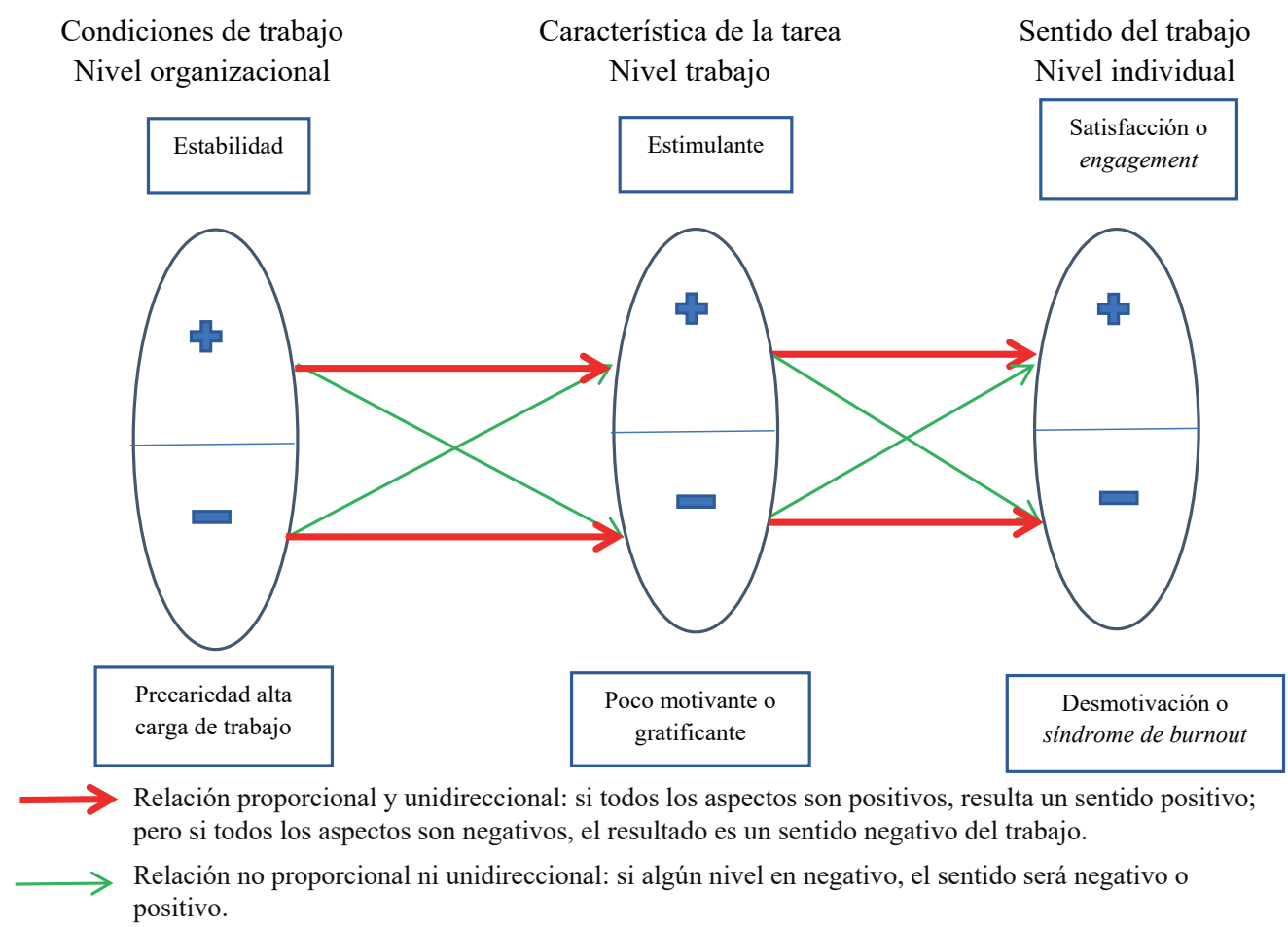

Figura 1. Sentidos del trabajo positivos o negativos como resultado de la relación entre las condiciones de trabajo y las características de la tarea (elaboración propia, basada en Romero, 2015).

\section{Método}

\section{Diseño}

Para la presente investigación se asumió un enfoque cuantitativo, con un diseño no experimental y de tipo descriptivo transversal (Hernández, Fernández \& Baptista, 2010), en donde se recolectaron y analizaron datos cuantitativos, se profundizó en la interpretación de los resultados, y se realizó un análisis estadístico de tipo descriptivo.

\section{Participantes}

Se realizó un muestreo intencional, por conveniencia, con los criterios de inclusión de sexo, edad, antigüedad en el centro, antigüedad en la profesión, tipo de contrato (estable o inestable) y tipo de universidad (pública o privada). En total, participaron 160 académicos pertenecientes a universidades de cinco ciudades de Colombia (Barranquilla, Bucaramanga, Cali, Santa Marta, Palmira), 78 hombres $(48.6 \%)$ y 82 mujeres (51.4\%), con una edad promedio de 33 años -con un rango de 23 a 65 años-, donde el mayor número de profesores se concentró en el intervalo de $33 \mathrm{a}$ 42 años (37.90 \%). En particular, el $48.4 \%$ del profesorado se encontraba vinculado contractual y laboralmente a universidades públicas, mientras que el $51.6 \%$ estaba en universidades privadas; $\mathrm{y}$, con respecto al tipo de contrato, el $53.3 \%$ se encontraba vinculado a su universidad con contrato permanente (tiempo completo), mientras que el $46.7 \%$ lo tenía de forma temporal (hora cátedra).

Por otra parte, según la cantidad de empleos que tenían, el $59 \%$ tenía un solo empleo -dedicación exclusiva al ejercicio académico en un solo centro universitario- y el $41 \%$ aseguró tener más de dos empleos en simultáneo, sin confirmar que todos los empleos obedecieran a la academia. Por otra parte, según la responsabilidad directiva, el 58.8 \% de los académicos encuestados respondió haber ejercido o tener responsabilidades directivas en sus trabajos como docentes universitarios; el $41.2 \%$ no había desempeñado ninguna actividad administrativa hasta el momento; respecto a la antigüedad en la profesión académica, el $43.7 \%$ llevaba entre 1 y 11 años en la profesión, el $38.4 \%$, entre 12 y 22 años, el $15.9 \%$, entre 23 y 33 años, y el $2 \%$, entre 34 y 44 años de antigüedad; y, finalmente, respecto a la antigüedad en el centro universitario, la mayoría estaba entre 1 y 9 años (76.2\%), seguida de entre 10 y 18 años (15.9\%), entre 19 y 27 años (4.6 \%) y entre 28 y 36 años $(3.3 \%)$ en la profesión.

\section{Instrumento}

Se aplicó el instrumento tipo cuestionario denominado QPW-5 (Cuestionario Proyecto Wonpum, quinta versión), diseñado para la recogida de datos del proyecto Working 
Under the New Public Management (WONPUM; Meanings, Risks \& Results), compuesto por 173 ítems distribuidos en diversas escalas, inventarios y algunas preguntas abiertas, aparte de la información sociodemográfica.

Específicamente, las escalas que mide el QPW-5 son: condiciones de trabajo (CT), carga de trabajo (CTP), bienestar laboral general (BLG), inventario de burnout de Maslach (MBI), engagement (UWES). El conjunto de las escalas de condiciones de trabajo del cuestionario fue diseñado y validado por Blanch, Sahagún y Cervantes (2010), al igual que la escala de bienestar laboral general (BLG), validada por Blanch, Sahagún, Cantera y Cervantes (2010). Las preguntas abiertas profundizaron cualitativamente en aspectos que habían sido tratados en las escalas, relacionados con el significado del trabajo. Cabe mencionar que el instrumento ha sido validado en investigaciones en población médica, donde se encontró que reproducía la estructura de los modelos teóricos de CT y BLG con alfas de Cronbach que oscilaban entre .80 y .97 para ambas escalas (Ochoa \& Blanch, 2016).

Asimismo, se han analizado las propiedades psicométricas del instrumento en población multiocupacional (psicólogos, médicos, abogados, gerentes, supervisores, entre otros), donde el instrumento mostró tener validez de constructo al ratificar la estructura factorial, así como con la confirmación del modelo de BLG como componente central de la dimensión psicosocial del trabajo (Millán,
Calvanese \& D'Aubeterre, 2013). En población universitaria colombiana (Morales, 2012), el instrumento mostró validez de contenido en las escalas de CT y BLG, además de confiabilidad en estas escalas con un alfa de Cronbach de .96 para ambas. En la Tabla 1 se presentan los índices de Cronbach obtenidos para la presente investigación.

\section{Procedimiento}

Para el desarrollo de la investigación, inicialmente se accedió a los académicos a través de personas clave en las universidades que respondieron a la convocatoria de participación en el estudio y que cumplieran con los criterios de inclusión; posteriormente, se aplicó el instrumento de manera voluntaria y confidencial -tras la firma del consentimiento informado-, para luego hacer tabulación de los datos en el programa estadístico SPSS 23; después, se hallaron las medias y desviaciones estándar, y, a partir de los datos obtenidos en la pregunta de las cinco palabras clave sobre la experiencia de trabajar, se realizó un análisis estadístico de orden categórico de las palabras, con el fin de cuantificarlas e identificar frecuencia de las mismas, y así realizar el análisis factorial de componentes principales.

Por otra parte, se realizó el análisis de contenido, con el que se hizo la sistematización de la información textual -con énfasis en el sentido del texto-(Bardin, 1996; Crespo, Bergére, Torregrosa \& Álvaro, 1998; Ochoa, 2012; Romero, 2015); y con los datos de las frecuencias de las palabras

Tabla 1.

Componentes cuantitativos del cuestionario e índices de Cronbach

\begin{tabular}{|c|c|c|c|c|}
\hline Nombre & Constructo & Rango tipo respuesta & $\begin{array}{l}\text { Psicométrico } \alpha \text { de Cron- } \\
\text { bach del modelo Wonpum }\end{array}$ & $\begin{array}{c}\text { Psicométrico } \alpha \text { de } \\
\text { Cronbach de la inves- } \\
\text { tigación }\end{array}$ \\
\hline $\begin{array}{l}\text { Escala de condiciones de } \\
\text { trabajo }\end{array}$ & $\begin{array}{l}\text { Condiciones de } \\
\text { trabajo percibidas }\end{array}$ & Likert de 0 a 10 & $\alpha$ entre .83 у .97 & $\alpha=.878$ \\
\hline Escala de carga de trabajo & $\begin{array}{l}\text { Carga de trabajo } \\
\text { percibida }\end{array}$ & Likert de 0 a 10 & $\alpha=.86$ & $\alpha=.724$ \\
\hline $\begin{array}{l}\text { Escala de bienestar laboral } \\
\text { general }\end{array}$ & $\begin{array}{l}\text { Bienestar y malestar } \\
\text { en el trabajo }\end{array}$ & $\begin{array}{l}\text { Dimensiones } 1 \text { y } 2 \text { : } \\
\text { diferencial semán- } \\
\text { tico de } 1 \text { a } 7 \\
\text { Dimensiones } 3 \text { a } 4 \text { : } \\
\text { Likert } 1 \text { a } 7\end{array}$ & $\alpha=.97$ & $\alpha=.93$ \\
\hline $\begin{array}{c}\text { Inventario de burnout de } \\
\text { Maslach }\end{array}$ & Burnout & Likert de 0 a 6 & En proceso de cálculo & $\alpha=.699$ \\
\hline Escala de engagement & $\begin{array}{c}\text { Engagement, impli- } \\
\text { cación en el propio } \\
\text { trabajo }\end{array}$ & Likert de 0 a 6. & En proceso de cálculo & $\alpha=.869$ \\
\hline
\end{tabular}

Nota. Elaboración propia. 
clave se procedió a realizar el análisis de los datos de las escalas de condiciones de trabajo (organización y método, organización y entorno, y organización y persona), bienestar laboral general (bienestar psicosocial y efectos colaterales), $M B I-G S$ (agotamiento, cinismo y eficacia) y engagement (vigor, dedicación y absorción), con el paquete estadístico R (Development Core Team, 2016) para el respectivo análisis estadístico de los datos.

Después, se utilizó la prueba de Shapiro Wilk (Ahad, Yin, Othman \& Yaacob, 2011; Patrício, Ferrerira, Oliveiros \& Caramelo, 2017; Razali \& Wah, 2011) para la confirmación de normalidad de los datos en las puntuaciones de las escalas estudiadas ( $\mathrm{p}<.05$, por lo cual se rechaza la hipótesis de normalidad); posteriormente, se empleó el coeficiente de correlación de Spearman para determinar las relaciones entre los puntajes de las dimensiones de las escalas; y, por último, se empleó el Análisis de Componentes Principales (ACP) para establecer el patrón de las dimensiones evaluadas entre las definiciones de trabajo percibidas por los participantes.

\section{Resultados}

A continuación se presentan los resultados obtenidos en el análisis de los datos recolectados en la aplicación del instrumento, en sus cinco escalas y preguntas abiertas, cuyo objetivo era mostrar la configuración del sentido de la profesión académica del colectivo de profesores encuestados.

Con respecto a la configuración del sentido de la profesión académica, en la Figura 2 se muestran las correlaciones a partir de un correlograma entre las puntuaciones de las dimensiones de las escalas estudiadas, en las cuales se definen -por color e intensidad en los diagramas circulares que aparecen en la parte superior derecha del gráfico- el grado de relación de la figura. Específicamente, se encontró que la correlación entre las dimensiones organización y método y organización y persona de la escala de condiciones de trabajo fue la más alta, con un de $\mathrm{r}=.78(\mathrm{p}=.000)$, seguida de la relación entre la dimensión efectos colaterales (bienestar laboral general) y la dimensión agotamiento (MBI-GS), con un valor de $\mathrm{r}=.76$ $(\mathrm{p}=.000)$. Adicionalmente, se encontraron 23 correlaciones que resultaron mayores de $\mathrm{r}=.40(\mathrm{p}<.05)$ o menores de $\mathrm{r}=-.40(\mathrm{p}=.05)$, algunas de ellas negativas, como el caso de la relación entre la dimensión agotamiento $(M B I-G S)$ y la de organización y método (condiciones de trabajo), con un $\mathrm{r}=.53(\mathrm{p}=.000)$, por lo cual, a mayor organización y método, menor agotamiento percibido por los participantes.

Por otra parte, el análisis de componentes principales (ACP) de las puntuaciones de las dimensiones evaluadas en el grupo de participantes mostró que los dos primeros componentes explicaron el $57.8 \%$ de la varianza total, $44.3 \%$ para el primer componente y $13.5 \%$ para el segundo (véase Figura 3 ).

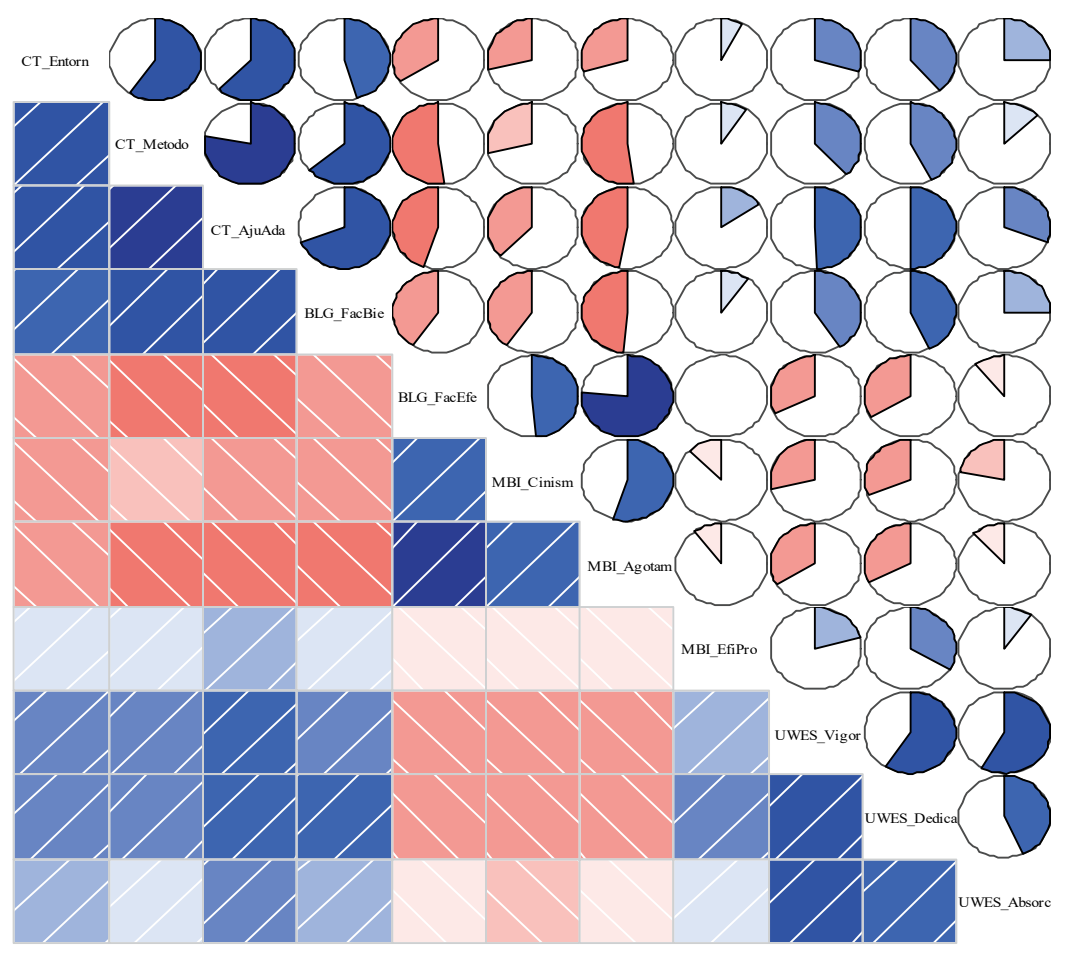

Figura 2. Correlograma entre las dimensiones de las escalas estudiadas años de edad. 
Como en el análisis correlacional, por medio del ACP también se evidenció que las dimensiones organización y método (.595), organización y persona (.780), organización $y$ entorno y bienestar psicosocial (.453) mostraron altas correlaciones entre ellas, con lo que se formó un grupo de cuatro variables; aunque en contraposición se encontraron las dimensiones cinismo (-.403), agotamiento (-.520) y efectos colaterales (-.486), lo que evidencia que este grupo de tres dimensiones se relaciona negativamente con el grupo formado de cuatro variables; es decir, que a un menor cinismo, agotamiento y efectos colaterales, mejores son las condiciones de trabajo y el bienestar psicosocial de los participantes $(p=.040)$. En un ángulo de casi $90^{\circ}$ entre los dos grupos de variables mencionados se encuentra el otro grupo, compuesto por las dimensiones vigor, dedicación, absorción y eficacia, por lo que se evidencia la no correlación con los otros dos grupos de variables.

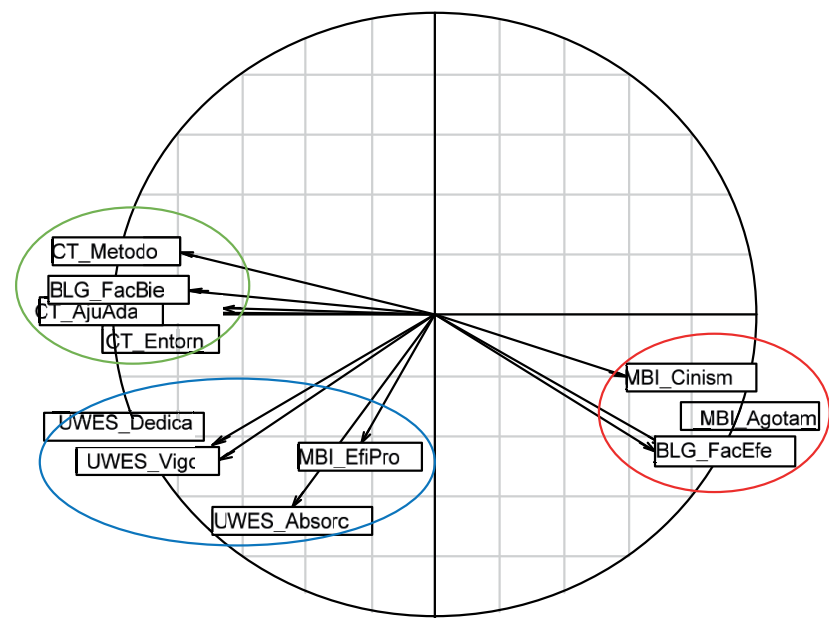

Figura 3. Círculo de correlaciones de los dos primeros componentes principales en la nube de puntos de las dimensiones estudiadas.

Adicional a esto, en la Figura 4 se muestran las subnubes de puntos de los participantes que describen en la quinta palabra un elemento positivo o negativo sobre su trabajo actual, en el plano de los dos primeros componentes principales -cabe resaltar que no se encontraron diferencias estadísticamente significativas en las primeras cuatro palabras que describen el trabajo-. Con esto se evidencia, a partir de la prueba Kruskal-Wallis, que existe una diferencia estadísticamente significativa en la primera componente (eje horizontal), entre los participantes que en su quinta palabra describieron el trabajo positivamente y los participantes que lo describieron negativamente $(P=.008)$. Este resultado indica que los docentes que describen el trabajo con una palabra negativa son los que presentan mayores puntajes en las dimensiones de cinismo, agotamiento y efectos colaterales, ya que en la nube de puntos se encuentran más hacia el lado derecho de la figura.

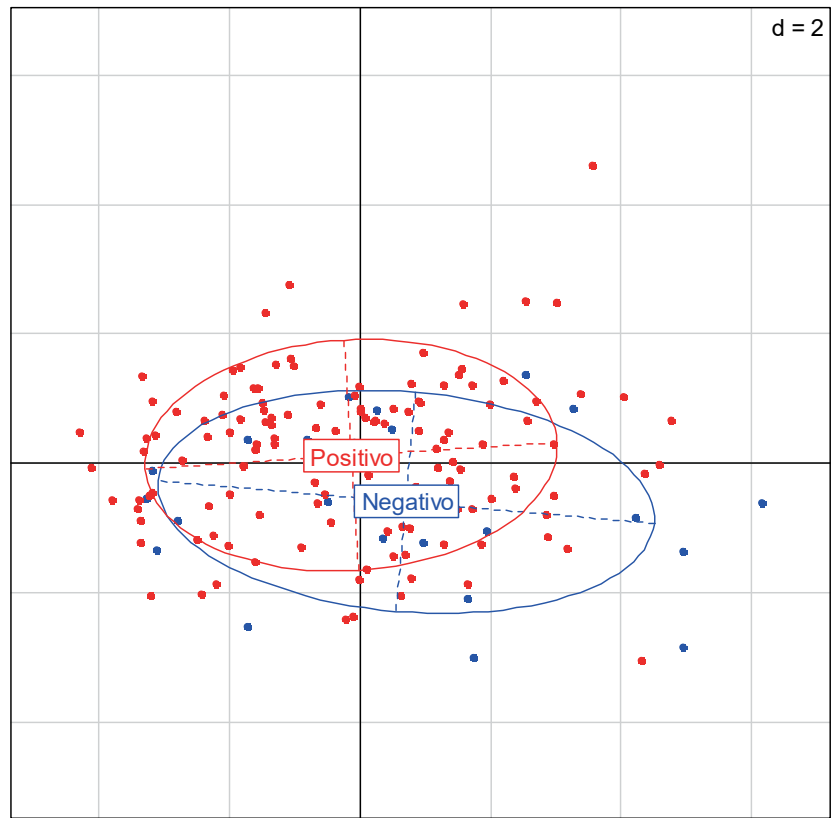

Figura 4. Proyección de los grupos de participantes que describen positivo o negativo el trabajo en su quinta palabra, en el plano de componentes principales.

Por último, dentro del conjunto de palabras que pueden destacarse como "negativas" para los participantes, se encuentran mala gestión, sobrecarga y malestar, tal como se observa en la Figura 5, con un $p=.049$.

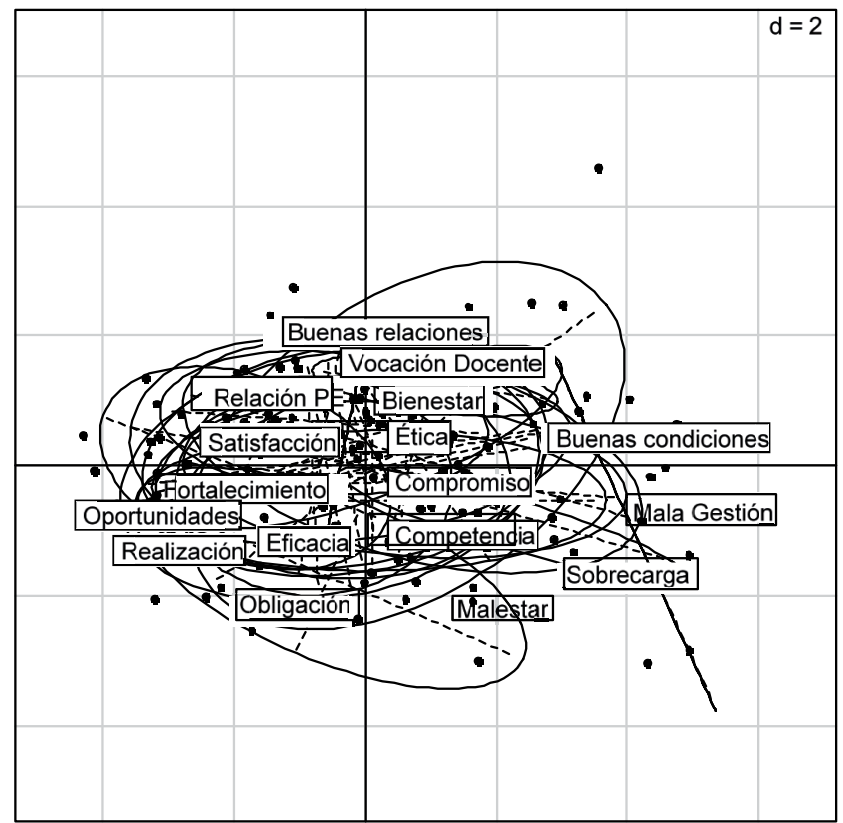

Figura 5. Proyección de los grupos de participantes que describen en palabras su trabajo, en el plano de componentes principales. 


\section{Discusión}

En el presente trabajo se indagó acerca de la configuración del sentido de la profesión académica en docentes universitarios ante las transformaciones de las condiciones de trabajo en las universidades colombianas. Los resultados obtenidos confirmaron la influencia que tienen las condiciones de trabajo en aspectos subjetivos de los individuos, como es el sentido de la profesión que se realiza (Blanch, 2011a, 2011b, 2013b; Ochoa \& Blanch, 2016; Romero, Blanch \& Rentería, 2016; Sisto, 2005, 2013). Específicamente, las dimensiones organización y método y organización y persona mostraron una relación significativa con la dimensión agotamiento de la escala MBI (Inventario de burnout de Maslach) en función con el ejercicio de la profesión académica, con aspectos como el entorno físico, el reconocimiento del trabajo realizado, la autonomía para la toma de decisiones, el tipo de contrato, la carga laboral, y la conciliación entre el trabajo y la vida privada. En este sentido, si las condiciones en las que se presenta el trabajo no son equilibradas, el agotamiento experimentado es mayor en los académicos.

Por consiguiente, se puede concluir que el sentido de la profesión académica se configura en términos que designan agotamiento por el trabajo debido a condiciones laborales fragmentadas que derivan de la temporalidad en la vinculación con las instituciones universitarias (Prieto et al., 2011; Romero, Blanch \& Rentería, 2016). A su vez, condiciones laborales como el aumento de responsabilidades para los profesionales de la academia -que obedecen al diseño de cargos "multitarea" en los centros universitarios, donde se combinan docencia, investigación y servicio social con tareas administrativas que se desglosan de la organización universitaria propiamente dicha-repercute en los académicos en efectos colaterales como el agotamiento (Garrido, Uribe \& Blanch, 2011; Sánchez \& del Sagrario, 2012).

Así entonces, es claro que las transformaciones en las universidades que se evidencian en los cambios en las condiciones de trabajo para los académicos generan un impacto en el sentido de la profesión académica, donde el bienestar de los docentes universitarios se ve deteriorado por la sobrecarga en las tareas a realizar en el trabajo académico. Para ejemplificar, algunas de las palabras que describen esta situación reportadas por los docentes universitarios fueron "mala gestión", "sobrecarga"y "malestar", las cuales pueden ubicarse en un plano negativo del sentido de la profesión (Romero, 2015), así como en su correlación con puntajes altos en las dimensiones de cinismo, agotamiento y efectos colaterales; esto permite concluir que los profesionales de la academia configuran el sentido de su profesión como un actividad desgastante y agotadora.

Por otro lado, es importante recalcar la naturaleza de las actividades relacionadas con la docencia que implican el profesionalismo de los docentes universitarios (Eirín et al., 2009; Garrido, Uribe \& Blanch, 2011; Nixon et al., 2010; Ochoa \& Blanch, 2016; Romero, Blanch \& Rentería, 2016), ya que con esto es posible entender que las palabras que significan su trabajo sean primero positivas y finalmente negativas, tal como se muestra en los resultados.

De este modo, la configuración del sentido de la profesión académica por parte de los docentes universitarios se decanta en los polos positivo y negativo (véase Figura 1; basada en Romero, 2015). El primero de ellos, entendido como una relación inversamente proporcional, en donde, a mejores condiciones de trabajo y un alto bienestar psicosocial, menor cinismo, agotamiento y efectos colaterales que percibidos del trabajo académico; y el segundo, el polo negativo, configura un sentido de la profesión negativo, en donde la relación proporcional entre condiciones de trabajo precarias, fragmentadas y de sobrecarga se configura con palabras negativas como malestar, mala gestión y sobrecarga. Acá, una de las dimensiones que se ve más afectada es la vocación docente, entendida como profesionalismo (Nixon et al., 2010; Romero, 2015; Romero, Blanch \& Rentería, 2016).

Se puede afirmar que la ambivalencia en la que se debate el profesorado académico colombiano parte de las condiciones cambiantes de las universidades, ya que estos cambios generan una resignificación del trabajo académico como elemento agenciador. Por esto se encuentra diversidad en las condiciones de trabajo según las funciones sustantivas de la universidad, es decir, que la configuración de los nuevos sentidos depende de la dedicación exclusiva a dictar clase o la realización de tareas administrativas, ser profesor investigador, profesor vocacional o académico; cambios contextuales y organizacionales en los que se envuelve la profesión.

Por otra parte, en la temporalidad del vínculo contractual como variable naturalizada de la flexibilización de las condiciones de trabajo académico, la presente investigación proporciona perspectivas interesantes para la exploración de la configuración del sentido de la profesión académica, con variables heterogéneas y dinámicas por medio de metodologías no solo cuantitativas, sino cualitativas y mixtas que tienden a formular el mejoramiento de las condiciones de trabajo del profesorado universitario, todo con el ánimo de minimizar los factores de riesgo psicosociales que hacen referencia a la organización universitaria -entendida como un sistema de trabajo con lógicas internas y globalizadas-. 
A manera de conclusión, se evidencia la relación entre las condiciones de trabajo de los profesionales de la academia, el bienestar laboral y el sentido que se configura de la profesión. Así, el contexto tanto global como nacional de las transformaciones de la academia denotan que el sentir respecto a la experiencia de trabajar en el ámbito académico presenta aspectos positivos -expresados como satisfacción y compromiso-y contrapuntos negativos - que se expresan como sobrecarga de trabajo y la precariedad contractual-.

De manera general, la información recolectada da la idea de las transformaciones de la academia en el ámbito colombiano y de su impacto en la vivencia del trabajo en el profesorado. Para futuros estudios, es importante tener en cuenta las tensiones latentes en los cánones de desarrollo de investigaciones y la posterior publicación de los resultados en revistas de alto impacto versus los tiempos de dedicación-donde se percibe un desequilibrio entre las actividades de docencia, investigación y vida familiar-, con el fin de obtener resultados que aporten a los procesos de desarrollo de políticas institucionales que propendan por el mejoramiento de acciones a favor del bienestar laboral y del balance trabajo-familia del profesorado universitario.

\section{Referencias}

Ahad, N. A., Yin, T. S., Othman, A. R., \& Yaacob, C. R. (2011). Sensitivity of normality tests to non-normal data. Sains Malaysiana, 40(6), 637-641.

Alcántara, S., \& Preciado, L. (2009). Consecuencias de las políticas neoliberales sobre el trabajo y la salud de académicos universitarios: el burnout como fenómeno emergente. Psicología y Salud, 19(2), 197-206. Recuperado de https:// www.uv.mx/psicysalud/psicysalud-19-2/19-2/SusanaMart\%EDnez-Alcantara.pdf

Anderson, G. (2008). Mapping academic resistance in the managerial university. Organization, 15(2), 252-270. doi: https://doi.org/10.1177/1350508407086583

Antunes, R., \& Alves, G. (2004). As mutações no mundo do trabalho na era da mundialização do capital. Educação e Sociedade, 25(37), 50-65. Recuperado de http://www.scielo.br/pdf/es/v25n87/21460.pdf

Ardichvili, A., \& Kuchinke, P. (2009). International perspectives on the Meanings of Work an Working: Current Research and Theory. Advances in Developing Human Resources, 11(2), 155-167. doi: https://doi.org/10.1177/1523422309333494

Arellano, D., \& Cabrero, E. (2005). La Nueva Gestión Pública y su teoría de la organización: ¿son argumentos antiliberales? Justicia y equidad en el debate organizacional público. Gestión y política pública, XIV(3), 599-618. Recuperado de https://www.redalyc.org/articulo.oa?id=13314307
Arenas, F., \& Andrade, V. (2013). Factores de riesgo psicosocial y compromiso (engagement) con el trabajo en una organización del sector salud de la ciudad de Cali, Colombia. Acta colombiana de Psicología, 16(1), 43-56. Recuperado de http://www.scielo.org.co/pdf/acp/v16n1/v16n1a05.pdf

Bardin, L. (1996). Análisis de Contenido. Madrid: Akal Ediciones.

Blanch, J. M. (2011a). Condiciones de Trabajo y Riesgos Psicosociales bajo la Nueva Gestión. Formación Continuada a Distancia (FOCAD). Madrid: Consejo General de Colegios Oficiales de Psicólogos de España.

Blanch, J. M. (2011b). Afrontando la nueva gestión pública: obedeciendo y resistiendo. En M. C. Ferrerira et al. (Orgs), Dominação e resistência no contexto trabalho-saúde (pp. 81-98). São Paulo: Mackenzie.

Blanch, J. M. (2013a). El trabajo académico digital como factor de riesgo psicosocial. Usos y abusos de las TIC en la educación superior. En L. Bianchetti \& T. M. Hetkowski (Eds.), Dossiê: Novas tecnologias, formação docente e pós-graduação. Número monográfico. Educação em Perspectiva. 4(2), 511-532. Recuperado de https://ddd.uab.cat/pub/ artpub/2013/128500/2013_Blanch_Trabajo_Academico_ Digital.pdf

Blanch, J. M. (2013b). La transición universitaria del claustro a la empresa. Tensiones éticas suscitadas por la gestión neoliberal. En Rezer, R. (Org). Ética e Ciência nas diferentes dimensões da Educação Superior: Experiencias, Reflexões e Desafios (pp. 87-108). Chapecó, Brasil: Argos.

Blanch, J. M. (2014). Calidad de vida laboral en hospitales y universidades mercantilizados. Papeles del psicólogo, 35(1), 40-47. Recuperado fr http://www.redalyc.org/articu lo.oa?id=77830184006

Blanch, J. M., Sahagún, M. A., \& Cervantes, G. (2010). Estructura factorial de la Escala de Condiciones de Trabajo. Revista de Psicología del Trabajo y las Organizaciones, 26(3), 175-189. Recuperado de http://scielo.isciii.es/scielo. php?script $=$ sci_arttext\&pid=S1576-59622010000300002

Blanch, J. M., Sahagún, M. A., Cantera, L., \& Cervantes, G. (2010). Cuestionario de Bienestar Laboral General: Estructura y Propiedades Psicométricas. Revista de Psicología del Trabajo y las Organizaciones, 26(2), 157170. Recuperado de http://www.redalyc.org/articulo. oa? id=231316500007

Brunner, J. (1990). Educación superior en América Latina. Cambios y desafios. Santiago de Chile: Fondo de Cultura Económica.

Brunner, J. (2012). La Universidad: comunidad de mercado o posmoderna? Bordón, 64(3), 27-38. Recuperado de https:// recyt.fecyt.es/index.php/BORDON/article/view/22033

Brunner, J. (2014). Transformación de lo público y el reto de la innovación universitaria. Bordón, 66(1), 4560. Recuperado de https://dialnet.unirioja.es/servlet/ articulo? codigo $=4626467$ 
Caballero, M. F., \& Nieto, L. E. (2015). Nueva gestión pública en Colombia y Binestar laboral del profesorado universitario. Entramando, 2(1), 124-134. Recuperado de http:// www.scielo.org.co/pdf/entra/v11n1/v11n1a09.pdf

Calera, A., Esteve, L., Roel, J. M., \& Uberti-Bona, V. (2014). La salud laboral en el sector docente. Guía para la prevención de riesgos. Recuperado de http://www.istas.ccoo.es/ descargas/docente.pdf

Castro-Gómez, S., \& Guardiola, O. (2002). Globalización, universidad y conocimientos subalternos: Desafíos para la supervivencia cultural. Nómadas, 16(1), 183-191. Recuperado de https://www.redalyc.org/articulo.oa?idp=1\&id=1051179 $41015 \&$ cid $=76040$

Chomsky, N. (13 de marzo de 2014). El Neoliberalismo tomó por asalto a las universidades. El Espectador. Recuperado de http://www.elespectador.com/noticias/educacion/elneoliberalismo-tomo-asalto-universidades-noam-chom sk-articulo-480438

Crespo, E., Bergère, J., Torregrosa, J., \& Álvaro, J.L. (1998). Los significados del trabajo: un análisis lexicográfico y discursivo. Sociología del Trabajo, 33, 51-70. Recuperado de https://dialnet.unirioja.es/servlet/articulo? codigo $=59401$

Development Core Team. (2016). A Language and Environment for Statistical Computing (version 3.4.0). Vienna, Austria: $\mathrm{R}$ Foundation for Statistical Computing. Recuperado de http://www.r-project.org

Eirín, R., García, H., \& Montero, L. (2009). Desarrollo profesional y profesionalización docente, Perspectivas y problemas. Profesorado, la revista de currículum y formación del profesorado, 13(2), 1-13. Recuperado de https://www. redalyc.org/articulo.oa?id $=56711798016$

Evans, L., \& Cosnefroy, L. (2013). The dawn of a new professionalism in the French academy? Academics facing the challenges of change. Studies in Higher Education, 38(8), 1201-1221. doi: https://doi.org/10.1080/0307507 9.2013.833024

Fernández, N., \& Pérez, C. (2011). La profesión académica universitaria en América Latina, en perspectiva comparada. Educação, Revista do centro de Educação, 36(3), 351-363. Recuperado de http://www.redalyc.org/ pdf/3845/384539805006.pdf

Garrido, J., Uribe, A., \& Blanch, J. M. (2011). Riesgos psicosociales desde la perspectiva de la calidad de vida laboral. Acta Colombiana de Psicología, 14(2), 27-34. Recuperado de https://www.redalyc.org/articulo.oa?id=79822611003

Guerrero, O. (2006). El mito del nuevo "Management" Público. Gerencia, 9(25), 1-54.

Hackman, J., \& Oldham, G. (1974). The job diagnostic survey: An instrument for the diagnosis of job and the evaluation of job redesign projects. New Haven: University of Yale.

Hackman, J., \& Oldham, G. (1975). Development of the job diagnostic survey. Journal of Applied Psychology, 60(2), 159-170. Recuperado de http://www.jwalkonline.org/
upload/pdf/Hackman $\% 20 \% 26 \% 20$ Oldham $\% 20(1975) \% 20$ -\%20Development $\% 20$ of $\% 20$ the $\% 20 J D S . p d f$

Hernández, R., Fernández, C., \& Baptista, P. (2010). Metodología de la Investigación. Lima: McGrawHill.

Isola, N. J. (2013). Profesión académica en educación. Entre el compromiso, la política y la ciencia (1973-1976). Educação, 36(3), 393-401. Recuperado de https://www.re dalyc.org/html/848/84828784012/

Ministerio de Educación Nacional (MEN). (2013). Reformas a la Educación Superior en América Latina: Ecuador, Chile, México, Perú y Colombia. Memorias del Encuentro. Bogotá, 23 de Octubre de 2013. Recuperado de http:// www.mineducacion.gov.co/1621/articles-340678_recur so_1.pdf

Mendoza, P., \& Berger, J. (2006). Capitalismo académico y la cultura académica: un estudio de caso. Recuperado de http:// web.missouri.edu/ mendozamp/EPAA_v16n23.pdf

Millán, A., Calvanese, N., \&, D'Aubeterre, M.E. (2013). Propiedades psicométricas del Cuestionario de Condiciones de Trabajo (qCT) en una muestra multiocupacional venezolana. Revista CES Psicología, 6(2), 28-52. Recuperado de https://www.redalyc.org/articulo.oa?id=423539422004

Mollis, M. (2003). Presentación. En: Mollis, M. (Comp), Las universidades en América Latina ¿reformadas o alteradas? La cosmética financiera (pp.9-15). Buenos Aires: CLACSO.

Morales, M. (2012). Prácticas de Resistencia docente en la universidad orientada al mercado. Capturas y fugas académicas en el contexto Colombiano (tesis sin publicar). Universidad Autónoma de Barcelona, España.

Morales, M., \& Blanch, J. M. (2013). Universidad posfordista y nuevas subjetividades docentes. ALAST. VII Congreso Latinoamericano de Estudios del Trabajo, São Paulo, Brasil. Recuperado de http://congressoalast.com/wp-content/ uploads/2013/08/253.pdf

Nixon, J., Marks, A., Rowland, S., \& Walker, M. (2010). Towards a new academic professionalism: a manifesto of hope. British Journal of Education, 22(2), 12011221. Recuperado de https://www.tandfonline.com/doi/ abs/10.1080/01425690124202

O'Reilly, D., \& Reed, M. (2011). The grit in the Oyster professionalism, mangerialism and leaderlism as discourses of UK public services. Organization Estudies, 32(8), 10791101. doi: https://doi.org/10.1177/0170840611416742

Ochoa, P. (2012). Significado del Trabajo en la Profesión Médica. Un Estudio Iberoamericano (tesis doctoral no publicada). Universitat Autónoma de Barcelona, España.

Ochoa, P., \& Blanch, J. M. (2016). Work, malaise, and wellbeing in Spanish and Latin-American doctors. Revista de Saúde Pública, 50, 21. Recuperado de http://www.scielo.br/ scielo.php?pid=S0034-89102016000100214\&script $=$ sci abstract\&tlng=es 
Patrício, M., Ferreira, F., Oliveiros, B., \& Caramelo, F. (2017). Comparing the performance of normality tests with ROC analysis and confidence intervals. Communications in Statistics-Simulation and Computation, 46(10), 7535-7551. doi: https://doi.org/10.1080/03610918.2016.1241410

Prieto, M. T., Valencia, A. C., Carrillo, J. C., \& Castellanos, J. A. (2011). Docentes: entre el malestar y la incertidumbre. En Pérez Mora, R., \& Monfredini, I. (Coords), Profesión académica: mecanismos de regulación, formas de organización y nuevas condiciones de producción intelectual (pp.121-132). México: Universidad de Guadalajara.

Razali, N. M., \& Wah, Y. B. (2011). Power comparisons of Shapiro-Wilk, Kolmogorov-Smirnov, lilliefors and anderson-darling tests. Journal of statistical modeling and analytics, 2(1), 21-33. Recuperado de https://www.resear chgate.net/publication/267205556_Power_Comparisons of_Shapiro-Wilk_Kolmogorov-Smirnov_Lilliefors_and_ Anderson-Darling_Tests

Rizo, H. E. (2004). Evaluación del profesor universitario. Una visión institucional. Revista iberoamericana de educación, 34(4), 1-15. doi: https://doi.org/10.35362/rie3423005

Romero, M. (2015). Significado del trabajo y sentido de la profesión en la academia. Un estudio en el contexto colombiano (tesis doctoral). Universitat Autonoma de Barcelona, España. Recuperado de http://www.tdx.cat/bitstream/ handle $/ 10803 / 313236 / \mathrm{mprc} 1 \mathrm{de} 1$. pdf; sequence $=1$

Romero, M., Blanch, J. M., \& Rentería, E. (2016). Significado del trabajo y sentido de la profesión académica en el contexto flexible de la universidad en Colombia. En Grueso, M. (Ed), Organizaciones Saludables y procesos organizacionales e individuales. Comprensión y retos. Bogotá: Editorial Universidad del Rosario.

Salanova, M., \& Schaufeli, W. (2004). El Engagement de los empleados: un reto emergente para la dirección de los Recursos Humanos. Estudios Financieros, 261, 109138. Recuperado de https://dialnet.unirioja.es/servlet/ articulo? codigo $=1046747$

Salanova, M., \& Schaufeli, W. (2009). El Engagement en el trabajo. Cuando el trabajo se convierte en pasión. Madrid: Alianza.

Sánchez, M., \& Del Sagrario, F. (2012). La precarización del trabajo. El caso de los maestros de educación básica en América Latina. Revistas Latinoamericana de Estudios Educativos, 42(1), 25-54. Recuperado de https://www.re dalyc.org/pdf/270/27023323003.pdf

Sisto, V. (2005). Flexibilización Laboral de la Docencia Universitaria y la Gestación de la Universidad sin Órganos. Un análisis desde la Subjetividad Laboral del Docente en Condiciones de Precariedad. En B. Levy \& P. Gentilli (Comp.), Espacio público y privatización del conocimiento. Estudios sobre politicas universitarios en América Latina (pp. 523-54). Buenos Aires: CLACSO.
Sisto, V. (2009). Cambios en el trabajo, identidad e inclusión social en Chile: desafíos para la investigación. Revista Universum, 24(2), 192-216. Recuperado de https:// scielo.conicyt.cl/scielo.php?script=sci_arttext\&pid $=\mathrm{S} 0718-23762009000200011$

Sisto, V. (2012). Identidades desafiadas: individualización managerialismo y trabajo docente en el Chile Actual. Psykhe, 21(2), 133-146. Recuperado de https:// scielo.conicyt.cl/scielo.php?script=sci_arttext\&pid $=$ S0718-22282012000200004

Sisto, V., \& López, V. (2014). Las voces y silencios de la Universidad hoy. La academia ante la transformación de la Universidad. Psicoperspectivas. Individuo y Sociedad, 13(1), 1-5. Recuperado de https://scielo.conicyt.cl/scielo. php?script=sci_arttext\&pid=S0718-69242014000100001

Slaughter, S., \& Leslie, L. (2001). Expanding and Elaborating the Concept of Academic Capitalism. Organization, 3(2), 154-161. Recuperado de https://journals.sagepub.com/doi/ pdf $/ 10.1177 / 1350508401082003$

Slaughter, S., \& Rhoades, G. (2004). Academic Capitalism and the New Economy: Markets, State and Higher Education. Baltimore: Johns Hopkins University Press.

Slaughter, S., \& Leslie, L. (1997). Academic Capitalism: Politics, Policies, and the Entrepreneurial University. Baltimore: The Jhons Hopkins University Press.

Spicer, A., \& Flemming, P. (2007). Intervening in the Inevitable: Contesting Globalization in a Public Sector Organization. Organization, 14(4), 517-541. doi: https:// doi.org/10.1177/1350508407078051

Spink, M., \& Medrado, B. (2013). Produção de Sentidos no Cotidiano: Uma abordagem teórico-metodológica para análise das práticas discursivas. En Spink, M. (Ed), Práticas Discursivas e Produção de Sentidos no Cotidiano. Aproximações teóricas e Metodológicas (pp. 22-41). Rio de Janeiro: Centro Edelstein de Pesquisas Sociais. Recuperado de http://maryjanespink.blogspot.com/2013/11/versao-vir tual-do-livro-praticas.html

Universia. (2015). Historia de las universidades: el marco medieval. Origen y consolidaciones. Recuperado de http:// universidades.universia.es/universidades-de-pais/his toria-de-universidades/historia-universidad-espanola/ marco-medieval/

Zabalza, M. A. (2013). Competencias docentes del profesor universitario. Bogotá: Narcea. Ediciones de la U.

Zamora-Fortuny, B. (2004). Profesionalismo y trabajo docente. Tempora, 7, 169-200. Recuperado de https://dialnet.unirio ja.es/servlet/articulo? codigo $=1420005$ 